\title{
Por una perspectiva kaingang sobre diabetes e hipertensión arterial: prácticas de autoatención, articulaciones y conflictos
}

\author{
SANDRA CAROLINA PORTELA GARCÍA
}

A partir de un acercamiento etnográfico a indígenas kaingang diagnosticados como hipertensos o diabéticos, en este artículo se describen prácticas de autoatención adoptadas por este grupo indígena para lidiar con dichas enfermedades y se explora cómo éstas forman parte de un entramado de relaciones entre las tradiciones médicas coexistentes en la región. A la luz de estas prácticas, se conocerán las perspectivas nativas sobre diabetes e hipertensión arterial, que difieren de lo establecido por la biomedicina y el Sistema Único de Salud. También se hablará de la autonomía de los pacientes indígenas y su capacidad de agencia para contestar las prácticas de biopoder dispuestas por el Estado mediante la práctica biomédica.

PALABRAS CLAVE: prácticas de autoatención, hipertensión, diabetes, kaingang, biopoder

Diabetes and Hypertension from a Kaingang Perspective: Self-Care Practices, Articulations and Conflicts

Based on an ethnographic approach among Kaingang Indians diagnosed as hypertensive or diabetic, this article describes different practices of selfattention adopted by this indigenous group with the intention of dealing with these diseases, and explores how they integrate for a complex network of relationships established among various medical traditions coexisting in the region. Within these practices, we will know the native perspectives on diabetes and hypertension that arise in this context and differ from the one established by biomedicine and the Brazilian Unified Health System. Likewise, through these practices, we will address the autonomy of indigenous patients and their agency capacity to respond biopower practices established by the State through biomedical practice.

Sandra Carolina Portela García

Universidad Externado de Colombia, Bogotá, Colombia

sandra.portela@uexternado.edu.co
KEYWORDS: self-attention practices, Kaingang, hypertension, diabetes, biopower 
on base en un acercamiento etnográfico y el acompañamiento a indígenas kaingang diagnosticados en algún momento de su vida como hipertensos o diabéticos por la biomedicina, el interés central de este artículo es describir las prácticas de autoatención adoptadas por este grupo para lidiar con estas enfermedades y conocer cómo éstas forman parte de un complejo entramado de relaciones entre las tradiciones médicas coexistentes en la región. Hablaremos de las concordancias y conflictos que desencadena la utilización de plantas curativas en té, tinturas y otras preparaciones, el consumo de medicamentos y las oraciones, rezos y benzimentos ${ }^{1}$ se observarán también las dinámicas de reapropiación y resignificación de los saberes sobre salud presentes en el territorio, para dar lugar a una perspectiva nativa sobre diabetes e hipertensión arterial (HTA) que difiere de manera sensible de las conceptualizaciones de la biomedicina y los programas de salud pública implementados por el Sistema Único de Salud (sus). Al mismo tiempo, las prácticas de autoatención utilizadas por los kaingang de la región para atender estas dos enfermedades nos llevarán a vislumbrar y analizar los espacios de autonomía de los pacientes indígenas y su capacidad de agencia para contestar las prácticas de biopoder establecidas por el Estado por medio de la práctica biomédica.

Para el desarrollo del eje analítico de este artículo, hago uso del concepto de prácticas de autoatención, en el sentido propuesto por Menéndez (2003), es decir, aquellas prácticas que buscan prevenir, dar tratamiento, controlar, aliviar o curar una enfermedad o padecimiento determinados, sin perder de vista que la autoatención se relaciona tanto con las condiciones técnicas y científicas, como con las religiosas, étnicas y políticas de una sociedad. La ventaja de este concepto es que contempla el contexto del que provienen las prácticas escogidas por los pacientes, mientras permite observar los usos articulados que surgen entre ellas (Menéndez, 2003; Garnelo y Wright, 2001). La perspectiva de Menéndez reconoce la diversidad de recursos terapéuticos que coexisten en Sudamérica y el dinamismo de los grupos sociales que se reapropian y resignifican elementos exógenos o propios disponibles, esto es, lo que entendemos como un contexto de intermedicalidad

1 En traducción libre al español, "bendiciones". 
(Follér, 2004). Asimismo, facilita el examen de la existencia de espacios de autonomía relativa de los pacientes, quienes, al usar determinada práctica de autoatención, no necesariamente pasan por la consulta y aprobación del especialista, sino que se permiten combinar prácticas que podrían parecer incompatibles o radicalmente contradictorias desde el punto de vista de los saberes que las originan (Menéndez, 2003).

Entre las categorías analíticas, nos gustaría indicar que la Tierra Indígena Xapecó (TIX) se considera un contexto de intermedicalidad (Greene, 1998; Follér, 2004), es decir, un lugar en el que a lo largo de la historia se han desarrollado contactos o relaciones intraétnicas e interétnicas, en el que coexisten varias tradiciones médicas, entre las que hay oposiciones y conflictos, así como reapropiación y resignificación de elementos y técnicas.

La investigación se realizó con indígenas kaingang de la aldea sede diagnosticados como hipertensos o diabéticos en algún momento de su vida, con independencia de su autorreconocimiento o no en el momento del encuentro etnográfico. El interés en el estudio de la diabetes y la HTA obedece a la preocupación generada en el área de salud pública en Brasil por la transición epidemiológica que enfrenta la población indígena en las últimas décadas, en la que la presencia de enfermedades crónicas no transmisibles es predominante. Además de la HTA y la diabetes, destacan el uso abusivo de sustancias, la obesidad y la desnutrición (Coimbra y Santos, 2000; 2001; Joe y Young, 1993).

En este trabajo se parte de la idea de que se teje una relación asimétrica entre la medicina tradicional kaingang y la biomedicina cuando interactúan (Oliveira, 1996; Diehl, 2001), ${ }^{2}$ y se pone en evidencia que esta última ignora las maneras particulares de las poblaciones para explicar y atender sus padecimientos (Menéndez, 2003).

Es importante señalar que, a partir de este tipo de problema y ante la demanda constante de los pueblos indígenas, éstos fueron reconocidos como culturalmente diferenciados por el Estado brasileño y más tarde se estableció una política de atención diferenciada en salud, con la intención de propiciar una atención que contemple sus particularidades. En un contexto de intermedicalidad, de relaciones asimétricas y de implantación de una política de atención diferenciada, se desarrolló esta investigación.

\section{HTA, diabetes y pueblos indígenas en Brasil: una mirada académica}

A pesar del reconocimiento del aumento de la prevalencia de diabetes y HTA entre pueblos indígenas en Brasil, puede decirse que existe una relativa escasez de estudios al respecto y que la mayoría se ha producido desde la perspectiva de la epidemiología, en particular sobre la HTA. De manera general, estos trabajos se han dedicado a establecer los índices de prevalencia de estas enfermedades en varias regiones del país y entre varias etnias (Cardoso, Mattos y Koifman, 2003; Gimeno et al., 2007; Salvo et al., 2009; Oliveira et al., 2011; Rocha et al., 2011; Bresan, Bastos y Leite, 2015), ${ }^{3}$ lo que reafirma la importancia de este problema de salud para los pueblos indígenas brasileños. Como mencionan Bresan, Bastos y Leite (2015), los datos arrojados por estos estudios aún son insuficientes para conocer de forma amplia la distribución y ocurrencia de estas enfermedades en el país.

Dichos estudios hacen hincapié en el contacto con la sociedad nacional como uno de los factores fundamentales que estimulan la presencia de estas

2

Sin embargo, como recuerda Menéndez (2003), también existen relaciones asimétricas entre medicinas indígenas y otras.

3 Para una lista de artículos referentes al tema, véanse Filho et al. (2015) y Buchillet (2007). 
enfermedades entre los pueblos indígenas a los que se aproximan: cambios en la alimentación y dinámicas de trabajo, entre otros, tendrían un papel esencial en el aumento de la HTA y la diabetes en estas comunidades. Este reconocimiento es necesario en la medida en que pone en evidencia la importancia de los procesos históricos, sociales y culturales que influyen en la salud de los pueblos indígenas (Coimbra y Santos, 2000), así como los cambios producidos por el contacto con la sociedad nacional en diferentes momentos históricos (Langdon y Rojas, 1991). Sin embargo, aunque estos factores se enuncian, no siempre se establece una correlación con el contexto particular del que se habla, debido a que los datos que componen estos estudios son principalmente fisiológicos y antropométricos.

En el caso específico de los indígenas kaingang, la literatura sobre HTA y diabetes no es muy diferente. En 2015, un estudio epidemiológico concluyó que hay $35 \%$ de prevalencia de dicha enfermedad en la región, cuando es un problema de salud expresivo en la localidad (Bresan, Bastos y Leite, 2015).

Por su parte, la antropología de la salud brasileña contemporánea ha realizado un esfuerzo sistemático y colectivo para pensar en la salud indígena, sus desafíos, avances y dificultades, ${ }^{4}$ desde una perspectiva en la que se privilegian los tratamientos etnográficos que atienden los puntos de vista nativos. Este enfoque etnográfico ha sido una estrategia primordial en el diálogo interdisciplinario con las ciencias de la salud y la salud colectiva, se han logrado avances en materia de salud indígena y se ha aportado a la construcción de políticas públicas que permiten una aproximación más adecuada en términos de reconocimiento de las particularidades culturales y contextuales de los pueblos para la atención de sus problemas de salud (Garnelo, Macedo y Brandão, 2003; Garnelo y Langdon, 2004).

Sin embargo, a pesar de la abundante literatura sobre la relación entre antropología y salud indígena en clave etnográfica y contexto interdisciplinar, en lo que se refiere a la diabetes y la HTA son rarísimos los trabajos que toman como eje de sus inquietudes las enfermedades. En líneas generales, éstas aparecen como consecuencias de otros factores y problemas de salud, como el consumo de alcohol, los hábitos alimenticios, etc. En ese sentido, esta investigación es una apuesta por aproximarse a estas enfermedades como núcleo de la investigación, incorporar la perspectiva antropológica y etnográfica, y ampliar la dimensión del problema al traer voces y perspectivas diversas a colación.

Al tornarse un tema periférico, es difícil encontrar literatura especializada en diabetes y HTA en Brasil que dé testimonio de las percepciones emic sobre dichas enfermedades entre los pueblos indígenas y grupos étnicos que componen el complejo tejido social brasileño. Permanece el vacío de conocimiento sobre estas enfermedades crónicas no transmisibles que, como muestra el acercamiento epidemiológico, son un problema relevante en la salud de los pueblos indígenas del país.

En esta ausencia sistemática, destaca el trabajo de Schweickardt y Garnelo (2003), quienes enfocan su mirada en las historias de vida de los pacientes hipertensos en Manaos, Amazonas, y observan que por medio de su subjetividad y capital simbólico es posible entender la no adhesión a los programas del Estado para la atención a estas enfermedades. El problema se estudia en una dimensión multivocal, para comprender la enfermedad más allá de los conocimientos biomédicos y la experiencia fisiológica de los cuerpos.

En este sentido, la disertación de Portela (2010), que da origen al presente artículo, contribuye a llenar el vacío de información sobre el tema y puede constituir un aporte interesante para estimular la discusión y la relevancia de tomarlo

$4 \quad$ Para un panorama más amplio de la bibliografía producida al respecto en Brasil, véase Buchillet (2007). 
como objeto de investigación en las ciencias sociales y la salud indígena brasileña, así como para alentar reflexiones interdisciplinarias y colectivas que permitan hacer propuestas en términos de políticas públicas en salud indígena.

\section{Caminos metodológicos}

En la cotidianidad de la vida de la aldea y del puesto de salud, así como en los eventos de educación en salud destinados a pacientes diagnosticados con las enfermedades que nos ocupan y organizados por el equipo de salud indígena de la región, la observación participante fue una estrategia fundamental.

No obstante, las primeras aproximaciones al campo fueron difíciles pues las relaciones no se establecían de manera fluida. Un domingo, en el partido de fútbol en la cancha de la aldea, tomada de la mano de mi hija Violeta, que entonces cumpliría tres años de edad, me sorprendí al ver que muchos kaingang la saludaban por su nombre, ella les contestaba y les contaba que yo era su mamá. Luego de la presentación, los indígenas conversaban curiosos sobre la presencia de la "blanquita" en el preescolar indígena, al que ella asistía en los horarios en que yo intentaba encontrar respuestas a las preguntas planteadas como problema de investigación para mi maestría, en $2005 .{ }^{5}$ En esos espacios, los niños y familiares se aproximaban para conocer a Violeta y conversar con ella. A partir de la curiosidad de los kaingang por mi hija y de su capacidad de socialización con niños y familias indígenas, emergí socialmente como persona y empecé a ser acogida y reconocida como la mamá de Violeta. Esto abrió un amplio abanico de posibilidades de relación, al que quizá no hubiera podido acceder sola y que dio pie a entrevistas, acompañamiento de itinerarios terapéuticos y narrativas. En especial, me permitió establecer una relación cotidiana de convivencia con los kaingang. Durante los cuatro meses de trabajo de campo en la aldea, me concentré de manera sistemática en los casos de diez kaingang, siete mujeres y tres hombres, todos diagnosticados como hipertensos. Tres de ellos, dos hombres y una mujer, eran diabéticos. ${ }^{6}$ En las reuniones que se llevaban a cabo en el puesto de salud, al que asistía un promedio de 25 personas, se ampliaba el locus de interlocutores con quienes departía de manera más esporádica en lo cotidiano de la aldea. Las familias y redes de apoyo de los pacientes también fueron importantes para entender las elecciones terapéuticas, los significados y las articulaciones tejidas alrededor de la HTA y la diabetes en este contexto.

En el mismo ejercicio de conversación, diálogo y convivencia cotidiana, me acerqué a los profesionales del equipo de salud indígena y tuve acceso a narrativas sobre el tema que nos convoca, la salud indígena y la atención diferenciada.

Para contextualizar al lector sobre la discusión que propongo, considero que debo señalar algunos aspectos fundamentales de la cosmología kaingang porque sustentan nuestras reflexiones sobre las prácticas de autoatención en diabetes y HTA, y porque a partir de ellos es posible comprender de manera más amplia la relación entre salud y enfermedad en este grupo indígena.

\section{Notas etnográficas sobre los kaingang}

Los indígenas kaingang se localizan en la región Sur del Brasil, en los estados de São Paulo, Santa Catarina, Rio Grande do Sul y Paraná. Pertenecen a la familia lingüística jê (Maybury, 1979). En la actualidad, constituyen el grupo jê más numeroso del país, con cerca de 37400 individuos (IBGE,

5

Desde ese año hasta 2015 tuve la oportunidad de trabajar con indígenas kaingang de manera sistemática.

6 Uno de ellos es dependiente de la insulina. 
2010). La organización social kaingang se asocia a la existencia de mitades clánicas patriarcales, kaimé y kairu (Oliveira, 1996; Almeida, 1998; Diehl, 2001). Esta organización se basa en la uroxilocalidad y la patrilinealidad. Los seres de la naturaleza también ser clasifican de acuerdo con el dualismo kaingang (Haverroth, 1997).

El cuerpo kaingang se entiende como la extensión del dominio cosmológico de la naturaleza. Así, la persona se constituye por el cuerpo, y más en específico, por la relación cuerpo-espíritu, kuprig o kumbã. ${ }^{7}$ El espíritu puede salir del cuerpo - hã - , entonces éste se debilita y enferma (Carvalho, 2008). El cuerpo y el espíritu deben recibir cuidados. Ante la presencia de enfermedad, el tratamiento debe encaminarse a la restitución de la fortaleza corporal.

Según Carvalho (2008), entre los kaingang y otros pueblos indígenas brasileños el cuerpo no es una totalidad y el uso del concepto de corporalidad se adecua en la medida en que en estas sociedades se revela una dialéctica para la comprensión de un sujeto social, pues al mismo tiempo que se tiene una matriz de cuño individual — semen, sangre, espacio en la aldea, casa - se tiene un colectivo social — alma, nombre, papel- - Turner afirma que el cuerpo kaingang debe entenderse como un conjunto de sentidos que congrega los aspectos sociales, biológicos y culturales, y constituye una identidad social, la cual es producto y productora de la estructura social y la cosmología (citado en Carvalho, 2008). Esta investigación atiende este llamado y comprende el cuerpo kaingang en esa multidimensionalidad.

Para el tratamiento de la salud y la enfermedad, en la medicina tradicional ${ }^{8}$ kaingang existe una serie de especialistas nativos cuyos saberes estarían encaminados al restablecimiento de la salud. Destacan el kujá - hombre sabio-, los curanderos, las remedieras ${ }^{9}$ y los benzedeiros. ${ }^{10}$ Según Crépeau (2002), los kujá distinguen entre dos tipos de saberes: guiados y no guiados. Los saberes guiados corresponden a las prácticas asistidas por auxiliares no humanos, como monos, serpientes, aves y jaguares, entre otros.

Los animales auxiliares asisten al kujá en el tratamiento de los enfermos, indican qué enfermedad padece y el remedio que se usará, la posología y la dieta que debe seguir. Los guías también pueden ser santos del catolicismo popular, como Nuestra Señora Aparecida — patrona de Brasil—, san João María, el Divino Niño Jesús y el Divino Espíritu Santo. Los saberes no guiados incluyen una variedad de prácticas entre las que destacan el empleo de hierbas medicinales en diferentes preparaciones y prácticas propias de la biomedicina.

La aldea sede donde se hizo la investigación es la principal de la TIX, constituida por diez aldeas, concentra a la mayoría de la población indígena kaingang, pues cuenta con puesto de salud, instituciones educativas, iglesias pentecostales y de otras religiones, ${ }^{11}$ y es donde se desarrollan con mayor intensidad las actividades políticas de la comunidad. En 2015, se estimaba que tenía una población de 1500 habitantes (Ghiggi, 2015).

7 Carvalho (2008) se refiere al espíritu con la palabra kaingang kuprig. Veiga (2000) denomina al espíritu con la palabra kaingang kumbã.

8 Así denominada por los kaingang, la medicina tradicional hace referencia a todos los conocimientos que, aunque no le sean propios, reconoce como suyos. En este artículo se entiende que la tradición está en continuo movimiento, es dinámica y se nutre de momentos históricos y saberes, se actualiza y se modifica.

9 Por lo general, mujeres especialistas en el conocimiento de las plantas medicinales.

10 Benzedeiros viene del verbo portugués benzer y se traduce como bendecir o persignar. Los benzedeiros tienen entre sus funciones bendecir el remedio. Algunos sabedores pueden tener conocimientos referentes a varios tipos de especialidades.

11 La introducción de iglesias pentecostales en la tix a partir de la década de 1940 influyó de manera importante en las dinámicas sociales entre los kaingang, lo cual es muy evidente en la TIX. 


\section{Diabetes y HTA: enfermedades crónicas, episódicas y curables}

La diabetes y la HTA se categorizan como enfermedades crónicas no transmisibles en la literatura biomédica y bajo esa forma de conocimiento sobre la salud se hace el diagnóstico en la TIX. El equipo multiprofesional de salud indígena que trabaja en la localidad comenzó a registrar, a partir de la década de 1990, la presencia de estas enfermedades entre los kaingang, y desde entonces su incidencia se ha incrementado (Bresan, Bastos y Leite, 2015).

$\mathrm{Al}$ acompañar cada día a diabéticos e hipertensos, fue posible percibir que los kaingang asocian con frecuencia estas enfermedades a problemas que surgen en el núcleo familiar, en especial problemas conyugales y dificultades para la crianza o la manutención del hogar. En el aspecto social, estas situaciones se reconocen como causantes de la enfermedad entre las mujeres. Respecto a la diabetes, escuchar que esta enfermedad estaría asociada al consumo excesivo de sustancias como bebidas alcohólicas o cafeína era una constante. Estas percepciones apuntan a entender la enfermedad como un fenómeno que va más allá de las causas biológicas que la desencadenan y permiten observar que el cuerpo kaingang no es indiferente a lo colectivo, lo que reafirma la multidimensionalidad que afecta la salud indígena (Portela, 2010).

En general, los síntomas asociados a la HTA y la diabetes, reconocidos por la comunidad, son el dolor de cabeza y la "tontura", que sería un estado en el que las personas no tienen capacidad para "hacer las cosas bien", sus facultades físicas son limitadas o están alteradas, y ponen al individuo en un estado de "debilidad" corporal. ${ }^{12}$ En algunos casos, la hinchazón en piernas y brazos también se reconoció como síntoma de estas enfermedades.

En un primer momento, las personas que comenzaron a presentar estos síntomas tomaron alguna iniciativa para controlarlos. Medicamentos, oraciones, té de plantas medicinales, entre otras estrategias, fueron utilizados para superar su cuadro de malestar. La biomedicina apareció como una alternativa terapéutica cuando los recursos empleados se mostraron insuficientes. En estos casos, la visita al puesto de salud trajo consigo el diagnóstico de una o las dos enfermedades.

En otros casos, el diagnóstico tomó por sorpresa a los indígenas kaingang que lo recibieron, pues el motivo de la consulta médica era otro malestar — dolor de espalda, malestar estomacal, etc.- - El diagnóstico también llegó con la participación en jornadas de salud, pues al tomar la presión se le informaba al paciente de la "anormalidad" del resultado y se le solicitaba que retornara para realizar el proceso pertinente para reafirmar o descartar el diagnóstico, y someterse a exámenes con la intención de descubrir otras posibles afecciones, como la diabetes.

Con el diagnóstico, el médico y el equipo de salud daban al paciente una serie de recomendaciones que debía seguir a cabalidad, con la intención de obtener buenos resultados en el control de estas enfermedades. Por lo regular, se señala el uso de medicamentos y prácticas de autocuidado enfocadas al cambio de hábitos, como hacer ejercicio y modificaciones en la dieta. ${ }^{13}$ Sin embargo, es interesante notar que la obtención de un diagnóstico no necesariamente se torna una herramienta con peso suficiente para que el paciente se autorreconozca como portador de esas enfermedades. Los diagnósticos alcanzados en una consulta que procura atender un problema de salud no relacionado con la HTA o la diabetes, y su carácter inesperado, hacen que las personas lo cuestionen. Durante la

12 Recordemos que la debilidad puede dar pie a que el espíritu salga del cuerpo, lo que causa enfermedad. Las acciones a tomar deben tener como objetivo recuperar la fortaleza.

13 Las prácticas de autocuidado atienden a la lógica biomédica y se centran en la idea del cuidado y la responsabilidad individual del propio estado de salud. 
interacción con los kaingang observé que el rechazo al diagnóstico se argumenta con frecuencia con base en la inexistencia de malestar, ausencia de síntomas y la capacidad del individuo de realizar sus trabajos diarios sin contratiempos.

La percepción de la inexistencia de la enfermedad, a pesar del diagnóstico, hace que la persona analice la experiencia vivida y evalúe los factores que incidirían en el diagnóstico. Algunos kaingang mencionaban que en el proceder del médico había un problema de escucha, porque tal vez la persona que los había atendido no había prestado suficiente atención a los síntomas que le relataban, cuando los había, o no consideraba la perspectiva del paciente, que manifestaba sentirse bien y se veía a sí mismo como portador de buena salud.

En el sector de la población que fue a consulta por el desarrollo de síntomas asociados a HTA y diabetes — dolor de cabeza, tontura, hinchazón- observamos una tendencia de los kaingang que pasan por esta experiencia a reconocerse en el diagnóstico realizado por el equipo de salud indígena. Sin embargo, en los dos casos, vemos que las formas de entender y tratar la diabetes y la HTA varían entre ellos y en relación con el conocimiento biomédico. Para comenzar, cabe mencionar que varias personas entrevistadas con quienes tuve la posibilidad de interactuar manifestaron haber sido diagnosticadas en algún momento con estas enfermedades, pero no se reconocían como hipertensos o diabéticos, pues en algún momento de sus vidas lograron "curarse", según lo narraron, con prácticas de autoatención, sobre las que volveremos más adelante. Algunos aseguraron que esta enfermedad hacía apariciones repentinas y otros reconocieron su cronicidad en sus cuerpos y sus vidas. En este sentido, observamos que entre los kaingang de la localidad la diabetes y la HTA pueden ser crónicas, como indica la biomedicina, pero también curables y episódicas.

Me gustaría resaltar que estas percepciones de la enfermedad están íntimamente ligadas a la vivencia individual, que se constituye como proceso y experiencia (Langdon, 2005), y sólo a partir de estos dos aspectos los individuos consiguen explicar su malestar, y explorar y escoger las formas de autoatención que les ofrecen la posibilidad de cura, control o alivio de sus padecimientos.

\section{Prácticas de autoatención y sus usos articulados en relación con la diabetes y la HTA entre los kaingang de la TIX}

A continuación, a partir de la narrativa construida por indígenas kaingang, procuraremos adentrarnos en las prácticas de autoatención que se aplican en la comunidad para lidiar, controlar o curar dichas enfermedades.

\section{LUCIANA $^{14}$}

Con la muerte de su marido, además de cuidar a sus dos hijos pequeños, Luciana empezó a trabajar en el puesto de salud de la región, en una época en la que la atención médica de la aldea dependía casi de manera exclusiva de los pocos agentes indígenas de salud. Ella es remediera, hija de un importante sabedor kaingang. El dolor de la pérdida, el cuidado de sus hijos y su trabajo la mantenían estresada. Para ella, esa situación desencadenó su cuadro de HTA, explicación compartida por su entorno familiar y social.

Una vez diagnosticada y debido a su circulación entre el puesto de salud y el uso de plantas medicinales, empezó a hacer uso de medicamentos, infusiones y tés de hierbas para controlar la enfermedad.

14 Se utilizan seudónimos para garantizar la privacidad de los interlocutores. 
Al transitar de la iglesia católica a la pentecostal, ahora guiada por el Espíritu Santo, Luciana percibió la efectividad del "remedio" orado — en la actualidad, orado por ella misma - y poco a poco dejó de consumir los medicamentos que ofrecía el puesto de salud. Con el paso del tiempo, la promesa de cura tuvo efecto, por lo tanto, redujo el consumo de té a dosis ocasionales con carácter preventivo. Con los años, sin embargo, Luciana advirtió en su cuerpo señales de que la hipertensión estaba a punto de volver: palpitaciones, canseira ${ }^{15}$ y una sensación de debilidad.

En esos momentos, reflexionó y se preguntó cuál de sus responsabilidades no estaba bien atendida: ¿estaba cumpliendo con sus responsabilidades con Dios? ¿Con la iglesia? ¿Con su comunidad? ¿Con su familia?

Por lo general, este autoexamen ayuda a Luciana a entender sus fallas en sus funciones sociales y colectivas, y también sus faltas individuales. Con el autodiagnóstico, se encomienda a Dios, al Espíritu Santo y prepara los remedios a la manera tradicional, como lo aprendió de su padre y de las mujeres de su familia. Orado con mucha fe y con la iluminación del Espíritu Santo, el remedio cura su cuerpo, restablece su salud y la devuelve a las responsabilidades con su familia y su comunidad.

\section{ANA Y PEDRO}

Ana y Pedro fueron diagnosticados como hipertensos y diabéticos. Ella es tipo II, él es tipo I, es decir, debe consumir insulina para su tratamiento, pues su cuerpo no la produce. Como compañeros de vida, comparten también la atención que le dan a sus males. Sus malestares los llevaron a obtener un diagnóstico en el puesto de salud.

En el fogón de hierro, Ana coloca una olla con agua. Mientras conversamos, me explica que las hojas de guabiroba, moranguinho y bergamota, ${ }^{16}$

que perfuman el ambiente, tendrán como destino el agua hirviendo, que se transformará en remedio. ${ }^{17}$

Una vez en el agua, las hojas reposan. Un rato después, son pasadas a un termo. Mientras Ana cuida de la preparación, Pedro ceba yerba mate en la cuia $^{18}$ y espera el termo que Ana le lleva gentilmente. Sentados los tres, soy invitada a beber de la primera cuia. Me cuentan que el médico insiste en la necesidad de que abandonen el consumo de mate, pero el que bebemos es "rezado", un poderoso remedio que les ayuda a equilibrar su presión. El mate, que se sirve y bebe a voluntad durante el día, acompaña la dosis generosa de medicamentos que deben tomar para atender sus enfermedades.

Para Ana, dejar el vino fue un gran sacrificio. El médico le pidió que lo hiciera y ella confiesa que de vez en cuando bebe un sorbito para matar las ganas. Para Pedro, comenzar a inyectarse insulina fue algo muy difícil. Las mujeres de la casa se aproximan y no pierden la oportunidad de contarme en tono jocoso que Pedro parecía un niño regañado, que fue divertido verlo receloso y lloroso cuando la enfermera vino por primera vez a enseñarle a usar las inyecciones, cuando siempre fue un hombre tan recio. Hasta hoy, alguien debe ayudarle a aplicarlas, pues él confiesa que no es capaz de hacerse "eso" a sí mismo, tres veces al día.

Pedro manifiesta que las inyecciones le causan tremendo dolor y explica que sólo se aplica dos dosis diarias, pues en su experiencia con eso es suficiente, se siente bien y se ahorra un dolor al día. Ana dice

16 Campomanesia xanthocarpa, Rubus rosaefolius y Citrus bergamia, respectivamente.

17 Se emplean también chapéu de couro (Echinodorus grandiflorus), chuchu (Sechium edule), cavalinho (Equisetum giganteum), erva de bugre (Casearia Sylvestris S), cidreira (Melissa officinalis), amora branca (Morus alba), ajo (Allium sativum) y pata de vaca (Bahuginia variegata).

18 Cuenco en el que se bebe el mate. 
que algunos de los remedios que debe tomar no la dejan dormir, le causan ardor en el estómago y no vale tomarlos antes o después de comer. Por eso reduce o elimina la dosis anterior al sueño nocturno para descansar. Reclama que el médico no le ha resuelto el problema y se pregunta si no habrá un remedio mejor, que haga el efecto deseado y no le cause ese malestar.

Aprovecho y pregunto a Ana por las botellas llenas de hojas y ramas que cuelgan en una pared de la cocina. Responde que María, antigua auxiliar de salud de la aldea Pinhalzinho y remediera, las ha rezado para ellos y que forman parte de sus remedios para controlar la hipertensión y lidiar con la diabetes. Entre las bolsas llenas de medicamentos, las hojas, ramas y botellas que cuelgan de la pared, resalta en el espacio de la cocina una mesa en la que Nuestra Señora Aparecida, san Jõao María, velas, estampas de santos y rosarios, entre otros objetos, se organizan como un altar. Ana y Pedro, fieles devotos católicos, encomiendan su salud a estos santos.

\section{RAFAEL}

Rafael era diabético. Diagnosticado en el puesto de salud por sus constantes malestares, comenzó un tratamiento de medicamentos. Convencido de la importancia de los remedios tradicionales en la salud kaingang, buscó una remediera que le ayudara a hacer el remedio, pues él no tenía el conocimiento. Comenzó a beberlo con los medicamentos que le recetaron en el puesto de salud. Poco a poco, Rafael se sintió fortalecido y recuperado, por lo que se aventuró a dejar el medicamento, sin consentimiento médico, y se quedó sólo con el té que la remediera preparaba para él. Su experiencia fue satisfactoria. Siguió el tratamiento con remedios tradicionales kaingang durante dos años más. Al sentirse restablecido por completo, suspendió también el tratamiento de la remediera y volvió a su vida normal. En la actualidad, se reconoce como una persona sana, vital, llena de la fortaleza necesaria para cumplir con sus labores diarias. Se siente incómodo con la constante intervención de los profesionales del puesto de salud, quienes le insisten en que retome el tratamiento. Cuenta que se ha vuelto un problema ir al puesto de salud para conseguir una pastilla para el dolor de cabeza o cualquier otro malestar, por lo tanto, lo evita, pues no sabe por qué los profesionales no entienden su premisa fundamental: “¡no estoy enfermo! ¡Ni aunque el médico diga que sí!”.

\section{De articulaciones y conflictos}

Durante la investigación, fue posible identificar tres formas principales de autoatención que los kaingang de la aldea sede utilizan por lo regular para la atención de la diabetes y la HTA: medicamentos, remedio do mato ${ }^{19}$ o preparaciones de hierbas medicinales, oraciones y benzimentos. Estas formas de atención provienen de varios saberes médicos: la biomedicina, la medicina tradicional kaingang y las iglesias pentecostales de la región.

Estas formas de atención se usan de manera articulada, "sin necesidad de la intervención central, directa e intencional de curadores profesionales, aun cuando éstos pueden ser la referencia de la actividad de autoatención” (Menéndez, 2003: 198). Es decir, los tratamientos se escogen de manera relativamente autónoma. Observamos que los pacientes deciden cómo lidiar con la enfermedad, muchas veces en diálogo con sus núcleos familiares y redes de solidaridad. Por ejemplo, en el caso del uso de medicamentos, hay personas que siguen las indicaciones médicas y otras que los ingieren en determinadas cantidades y momentos, sin tomar en cuenta su uso "ideal", propuesto por la biomedicina y la

\section{9}

Del monte. 
farmacología, pues responden de manera coherente a las necesidades físicas y sociales que el paciente percibe en su experiencia corporal, en relación con el uso del medicamento y la enfermedad en general.

Acerca del uso de remedios del monte o infusiones, el paciente o uno de sus familiares cercanos pueden prepararlos, siempre que tengan el conocimiento. En otros casos, la preparación del té se encarga a terceros, entre los que se incluyen las remedieras u otros conocedores. Su uso puede acompañarse de rezos u oraciones sobre la preparación.

Es importante destacar que los remedios de la medicina tradicional kaingang son rezados después de su preparación, en especial por los curadores. Los rezos no tienen un esquema específico, cada curador tiene su forma particular de rezar el remedio. Los rezos se asocian a la praxis católica y al catolicismo popular, y pueden llegar a invocar uno o varios santos (Oliveira, 1996; Ghiggi, 2006). Se solicita su capacidad de agencia sobre el remedio, potenciado por su presencia. Hoy, con la influencia de las iglesias pentecostales, la elaboración de remedios con plantas ha incorporado otra serie de elementos y explicaciones que le dan validez a su continuación como forma de atención vigente entre los kaingang de la TIX. Luciana, remediera de la región, comenta que ella "ora" 20 el remedio para recibir las potencialidades del Espíritu Santo, ahora concebido en

Los kaingang de la región establecen una diferenciación importante en este sentido: "Ios católicos rezan, los evangélicos oran" (entrevista con Luciana, Terra Indígena Xapecó, 20 de octubre de 2009).

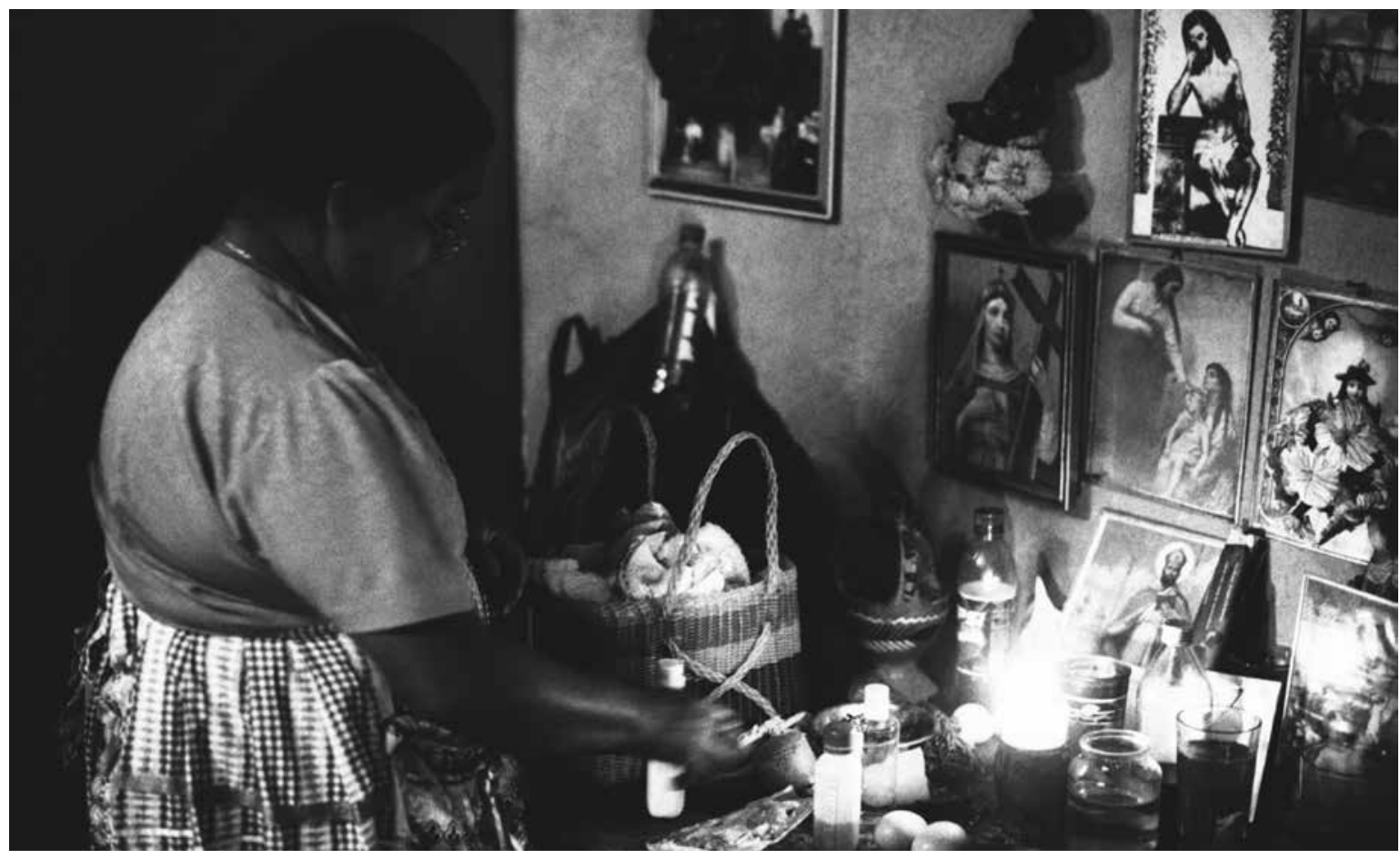

Ricardo Ramírez Arriola - La comadrona es una figura de respeto en los pequeños poblados. Además de atender partos, es conocedora de plantas, rituales y fases de la luna, asociados a la salud. Atiende la mayoría de los partos en las poblaciones indígenas y con un alto nivel de marginación. San Pedro Pinula, Jalapa, Guatemala, 2000. 
el sentido propuesto por la iglesia pentecostal, de la que forma parte.

Si nos adentramos en la relación entre salud e iglesias pentecostales, veremos que al presentarse casos de diabetes y HTA la iglesia pasó a ser una alternativa terapéutica, incluso para las personas que no pertenecen a ella. El paciente feligrés recurre a la oración de sus "hermanos" y del pastor de su iglesia, asiste a los cultos. En casos más difíciles, se ofrecen campañas ${ }^{21}$ por su mejoría. La enfermedad, en este contexto, puede entenderse como una prueba de fe, un llamado de atención por no cumplir con rigurosidad sus obligaciones con la iglesia y con Dios.

En el caso de las iglesias pentecostales, a pesar de que el paciente deba pedir primero el auxilio de la oración, es muy interesante que exista una gran aceptación de tratamientos biomédicos y remedios do mato tradicionales. Por ejemplo, en enfermedades como la diabetes, la acción de la oración y de la iglesia se encamina de manera explícita a ayudar en el plano espiritual, y se entiende que los remedios y medicamentos atienden el malestar corporal. Don Marino lo resume así: "los medicamentos [son] para el cuerpo y Dios para nuestra alma" (entrevista con Marino, Terra Indígena Xapecó, 30 de noviembre de 2009). En ese sentido, es interesante hacer una analogía con la noción de cuerpo kaingang propuesta por Carvalho (2008), en la que el restablecimiento de la salud o la obtención de la fuerza debe atender necesariamente cuerpo y espíritu, dupla indivisible para la salud kaingang. Cabe anotar que en la cotidianidad, con la alimentación y otras dinámicas sociales, se atiende la necesidad de nutrir el cuerpo, que también es espíritu, tanto individual como colectivo.

En relación con las prácticas de autoatención provenientes de la biomedicina, existe una cierta insatisfacción entre los profesionales de la salud, por la falta de adhesión o la resistencia de los indígenas kaingang a cumplir con el uso "ideal" de los medicamentos y prácticas de autocuidado que ellos les sugieren. Por el contrario, los kaingang hacen uso de medicamentos no sólo por su eficacia, sino también como parte de una reivindicación de sus derechos políticos, en este caso, el acceso a medicamentos y a la salud indígena y pública (Portela, 2010; Diehl, 2001). Dicho uso no está en desacuerdo con otras prácticas y saberes, como la medicina tradicional kaingang y los saberes médicos de las iglesias pentecostales. En este sentido, podemos proponer que este tipo de saberes se tornan más dinámicos y flexibles, capaces de incorporar y dialogar con otras prácticas sin que por ello se generen polémicas que prohíban su uso articulado.

Llama la atención el hecho de que los profesionales de la salud que actúan en la región logren empatía con las iglesias pentecostales, pues las reconocen como eficaces en tratamientos de alcoholismo y afirman que los diabéticos que pertenecen a ellas tienden a seguir un tratamiento biomédico de manera más estricta. Sin embargo, es paradójico que las iglesias no sean bien vistas por los profesionales, pues les incomoda que el paciente hipertenso evangélico suponga que ha sido curado, o que puede llegar a serlo, por la acción del Espíritu Santo y abandone el tratamiento biomédico.

A partir de los contextos y narrativas que hemos presentado, se observa que los kaingang de la TIX establecen diferentes relaciones con las tradiciones médicas, que coexisten y se tornan alternativas terapéuticas disponibles y vigentes para atender su salud. El encuentro de estas tradiciones implica la introducción de nuevos elementos, enfermedades y tratamientos que pueden ser aceptados, apropiados o rechazados por cada comunidad, en coherencia con sus tradiciones particulares (Greene, 1998; Langdon y Diehl, 2007; Garnelo y Wright, 2001). Ante esta gama de posibilidades, es frecuente que el paciente

Cadenas masivas y sistemáticas de oración, que se prolongan por semanas o meses. 
privilegie algunas alternativas en detrimento de otras o escoja prácticas que pueden parecer antagónicas o fuertemente diferenciadas (Ménendez, 2003).

Sin embargo, este antagonismo aparente muestra la pluralidad médica, la dinámica de las culturas - que con frecuencia se imaginan estáticas-, la capacidad de articulación de saberes médicos por parte de los individuos - por lo tanto, su capacidad social de agencia - y la existencia de espacios de relativa autonomía del paciente para lidiar con sus dolencias y malestares.

\section{Prácticas de autocuidado y relaciones de biopoder}

En páginas anteriores nos dedicamos a trazar el contexto de intermedicalidad de los kaingang de la TIX a partir de las prácticas de autoatención y destacamos sus usos articulados a favor de la atención a la diabetes y la HTA. Me gustaría apuntar que el conocimiento biomédico es también un agente importante en la TIX. Representado por el equipo de salud indígena que opera en la zona, centra su práctica en el autocuidado, que responsabiliza al individuo por el estado de su salud y elimina desde esta perspectiva el contexto social y cultural en el que el paciente se desenvuelve, así como los factores estructurales que impactan en su salud y calidad de vida. Respecto a la diabetes y la HTA, la práctica central de autocuidado establecida por la biomedicina y por los profesionales de salud indígena de la TIX, en este caso, es el uso de medicamentos que deben aplicarse o ingerirse de manera correcta, razón por la cual los esfuerzos se dirigen con frecuencia a explicar dosis, usos, posologías, etcétera.

Algunos focos problemáticos identificados por el equipo de salud de la aldea, para el control de la diabetes y la HTA, son la mala alimentación y la falta de actividad física, por ello se hace hincapié en orientar a los pacientes para que mantengan una alimentación saludable, es decir, en la que predomine el consumo de frutas y verduras, en detrimento de grasas, azúcares, alimentos industrializados, etc., y hacer más ejercicio.

Con la intención de orientar, acompañar y recordar la importancia de las indicaciones a sus pacientes, el equipo de salud hace una serie de actividades dirigidas a hipertensos y diabéticos en la TIX. La más importante es una reunión mensual de educación en salud, en el puesto de salud.

Durante el trabajo de campo, se observaron diferencias fundamentales en lo que se percibe como una buena alimentación para los profesionales y los kaingang. Los alimentos que los primeros proponen como saludables, de manera general, desde la perspectiva kaingang suelen estar en la categoría de alimentos fracos, ${ }^{22}$ cuyo consumo debilita el cuerpo y abre la posibilidad de enfermar, como se explica en la cosmología de este pueblo.

La lógica y objetivos de las actividades de educación en salud, estrategia central de varios programas implementados por el sus, como el Hiperdia, ${ }^{23}$ funcionan bajo "las premisas del proceso educativo, cuando éste presupone que los mensajes educativos definidos por el 'emisor-profesor' deben ser reproducidos literalmente por el 'receptor"' (Garnelo, 2001: 283). Es decir, se supone que el "otro", al que busca aproximarse, no posee conocimientos sobre su salud y su cuerpo, o que éstos son equivocados y deben ser reemplazados, lo que niega la especificidad cultural, histórica y social de la sociedad o grupo que trata.

Débiles. Otras formas de clasificación de los alimentos entre los kaingang fueron discutidas por Oliveira (2009).

23 Programa destinado al registro y acompañamiento de portadores de HTA y diabetes mellitus atendidos en el sus. Véase <http://datasus.saude.gov.br/sistemas-e-aplicativos/ epidemiologicos/hiperdia>, consultado el 25 de octubre de 2017. 
Los ejercicios de educación en salud con los pacientes hipertensos y diabéticos a quienes acompañamos durante el trabajo de campo son un reflejo de la práctica biomédica general, que busca no sólo la adherencia al tratamiento, sino motivar un cambio significativo en los hábitos y costumbres de las personas. Los ejercicios son invasivos, en la medida en que permean todos los espacios de la vida: alimentación, higiene, crianza, y hasta actividades económicas y productivas, que se sugieren de manera sistemática y con las cuales se establece una relación de biopoder clara y asimétrica sobre los cuerpos que intentan controlar y disciplinar. ${ }^{24}$

La continua imposición de una serie de prácticas de autocuidado demuestra la incapacidad del equipo de salud indígena que actúa en la región para articular sus saberes de manera dinámica con los de la medicina tradicional kaingang. Como hemos intentado mostrar, esta capacidad está presente entre los indígenas, quienes recurren a una diversidad de prácticas de autoatención combinadas, sin importar la aparente incoherencia que pueda existir entre los sistemas médicos que las producen (Menéndez, 2003). Este tipo de relación, que suele ser común en el sistema biomédico, acaba deslegitimando los conocimientos en salud de los pueblos indígenas y crea una paradoja - en especial en el contexto brasileño, en el que existe una política de atención diferenciada en salud indígena-, en la medida en que la particularidad cultural de estos conocimientos aparece en términos retóricos y legales como un diferencial que debe ser respetado y valorado.
Por último, quisiera destacar que el acercamiento etnográfico posibilitó la observación de las articulaciones entre prácticas de autoatención. Éstas no sólo dan testimonio de un pluralismo médico que dialoga, se negocia y renegocia sin cesar, sino que también, por medio de ellas, es posible trazar conexiones con prácticas cotidianas de los kaingang, como la alimentación y la corporalidad, entre otros aspectos que hablan de las cosmologías de los pueblos indígenas —en este caso, la íntima relación entre cuerpo y espíritu, debilidad y fortaleza, apropiaciones y resignificaciones de lo tradicional, etc. - y facilitan el acercamiento a las percepciones sobre el proceso de salud/enfermedad/ atención de las comunidades indígenas, al ejemplificar el dinamismo de su cultura y prácticas tradicionales (Menéndez, 2003).

A partir de la descripción de las prácticas de autoatención observadas en clave etnográfica y de la experiencia kaingang en relación con la diabetes y la HTA, vemos que el paciente se torna agente de su propia vida y su sociedad, rescata, aunque de manera parcial, la autonomía que le es cuestionada y retirada por las relaciones de biopoder que la biomedicina pretende ejercer sobre sus cuerpos y sus vidas. D

24 El biopoder se entiende como las maneras en que los Estados explotan numerosas y diversas técnicas para subyugar los cuerpos y controlar a la población (Foucault, 2006).

\section{Bibliografía}

\footnotetext{
Almeida, Ledson Kurtz de, 1998, Dinâmica religiosa entre os kaingang do posto indígena Xapecó-sc, tesis de maestría en antropología social, Universidade Federal de Santa Catarina, Florianópolis.

Bresan, Deise, João Luiz Bastos y Maurício Soares Leite, 2015, “Epidemiologia da hipertensão arterial em indígenas kaingang, Terra Indígena Xapecó, Santa Catarina, Brasil, 2013", en Cadernos de Saúde Pública, vol. 31, núm. 2, pp. 331-344.

Buchillet, Dominique, 2007, Bibliografía crítica da saúde indigena no Brasil (1844-2006), Abya Yala, Quito.
} 
Cardoso, Andrey, Inés Mattos y Rosalina Koifman, 2003, "Prevalência de diabetes mellitus e da síndrome de resistência insulínica nos índios guarani do estado do Rio de Janeiro”, en Carlos Avelardo Coimbra Jr., Ricardo Ventura y Ana Lucía Escobar (orgs.), Epidemiología e saúde, povos indígenas no Brasil, Fundação Oswaldo Cruz, Río de Janeiro, pp. 169-185.

Carvalho Rosa, Patrícia, 2008, "A noção de pessoa e a construção de corpos kaingang na sociedade contemporânea", en Espaço Ameríndio, vol. 2, núm. 1, pp. 15-43.

Coimbra, Carlos Avelardo Jr. y Ricardo Ventura Santos, 2000, "Saúde, minorias e desigualdades: algumas teias de inter-relações, com ênfase nos povos indígenas no Brasil”, en Ciência \& Saúde Coletiva, vol. 5, pp. 125-132.

-, 2001, "Perfil epidemiológico da população indígena no Brasil: considerações gerais", documento de trabajo 3, Universidade Federal de Rondônia-Escola Nacional de Saúde Pública, Porto Velho. Disponible en línea: <http://www.cesir.unir.br/pdfs/doc3.pdf>.

Crépeau, Robert, 2002, "A prática do xamanismo entre os Kaingang do Brasil meridional: uma breve comparação com o xamanismo Bororo", en Horizontes Antropológicos, vol. 8, núm. 18, pp. 113-129.

Diehl, Eliana Elisabeth, 2001, Entendimentos, práticas e contextos sociopoliticos do uso de medicamentos entre os kaingang (Terra Indigena Xapecó, Santa Catarina, Brasil), tesis de doctorado, Fundação Oswaldo Cruz, Río de Janeiro.

Filho, Zilmar Augusto de Souza, Alaidistânia Aparecida Ferreira, Bernardo dos Santos y Angela Maria Geraldo Pierin, 2015, "Prevalência de hipertensão arterial em indígenas do Brasil: uma revisão sistemática com meta-análise”, en Revista da Escola de Enfermagem, vol. 49, núm. 6, pp. 1016-1026.

Follér, Maj-Lis, 2004, “Intermedicalidade: a zona de contato criada por povos indígenas e profissionais de saúde”, en Esther Jean Langdon y Luiza Garnelo (orgs.), Saúde dos povos indígenas: reflexões sobre antropologia participativa, Associação Brasileira de Antropologia/ ContraCapa, Río de Janeiro, pp. 129-148.

Foucault, Michael, 2006, Historia de la sexualidad, vol. I: La voluntad de saber, Siglo XXI Editores, Barcelona.

Garnelo, Luiza, 2001, “Projeto Rede Autonoma de Saúde Indígena. Uma experiência de educação e comunicação em saúde indígena”, en Eymard Mourão Vasconcelos (org.), A saúde nas palavras e nos gestos, Hucitec, São Paulo, pp. 237-260.

Garnelo, Luiza y Esther Jean Langdon (orgs.), 2004, Saúde dos povos indígenas. Reflexões sobre antropología interpretativa, Associação Brasileira de Antropologia/ContraCapa, Río de Janeiro.

Garnelo, Luiza, Guilherme Macedo y Luiz Carlos Brandão, 2003, Os povos indígenas e a construção das políticas de saúde no Brasil, Organização Pan-Americana da Saúde, Brasilia.

Garnelo, Luiza y Robin Wrigth, 2001, "Doença, cura e serviços de saúde. Representações, práticas e demandas Baníwa”, en Cadernos de Saúde Pública, vol. 17, núm. 2, pp. 273-284.

Ghiggi Junior, Ari, 2006, Representações e práticas no tratamento de infortúnios entre os kaingang crentes da aldeia sede da Terra Indigena Xapecó, tesis de licenciatura en ciencias sociales, Universidade Federal de Santa Catarina, Florianópolis.

—_ 2015, Uma abordagem relacional da atenção à saúde a partir da Terra Indígena Xapecó, tesis de doctorado en antropología social, Universidade Federal de Santa Catarina, Florianópolis.

Gimeno, Suely Godoy Agostinho, Douglas Rodrigues, Heloisa Pagliaro, Eduardo Nozaki, Evandro Emilio de Souza Lima y Roberto Geraldo Baruzzi, 2007, "Perfil metabólico e antropométrico de índios aruák: Mehináku, Waurá e Yawalapití, Alto Xingu, Brasil Central, 2000/2002", en Cadernos de Saúde Pública, vol. 23, núm. 8, pp. 1946-1954.

Greene, Shane, 1998, "The Shaman's Needle: Development, Shamanic Agency, and Intermedicality in Aguaruna Lands, Peru”, en American Ethnologist, vol. 25, núm. 4, pp. 634-658.

Haverroth, Macir, 1997, Kaingang, um estudo etnobotânico. O uso e a classificação das plantas na Área Indígena Xapecó, tesis de maestría en antropología social, Universidade Federal de Santa Catarina, Florianópolis.

Instituto Brasileiro de Geografia e Estatística (IBGE), 2010, O Brasil indígena. Disponible en línea: <https://indigenas.ibge.gov.br/images/pdf/ indigenas/folder_indigenas_web.pdf>.

Joe, Jennie Rose y Robert Young, 1993, Diabetes as a Disease of Civilization: The Impact of Culture Change on Indigenous Peoples, Mouton de Gruyter, Nueva York.

Langdon, Esther Jean, 2005, "A construção social da doença e o seu desafio para a prática médica”, en Roberto Baruzzi y Carmen Junqueira (orgs.), Parque indígena do Xingu: saúde, cultura e história, Terra Virgem, São Paulo, pp. 115-131.

Langdon, Esther Jean y Eliana Diehl, 2007, "Participação e autonomia nos espaços interculturais de saúde indígena: reflexões a partir do sul do Brasil”, en Saúde e Sociedade, vol. 16, núm. 2, pp. 7-12. 
Langdon, Esther Jean y Blanca Rojas, 1991, "Saúde: um fator ignorado numa situação de mudança rápida. A situação da área indígena Ibirama (sc)", en Silvio Coelho dos Santos (org.), A barragem de Ibirama e as populações atingidas na área indígena", en Boletim de Ciências Sociais, vol. 51, pp. 65-89.

Maybury Lewis, David (org.), 1979, Dialectical Societes: The Gé and Bororo of Central Brazil, Harvard University Press, Cambridge.

Menéndez, Eduardo, 2003, "Modelos de atención de los padecimientos: de exclusiones teóricas y articulaciones prácticas", en Ciência \& Saúde Coletiva, vol. 8, núm. 1, pp. 185-208.

Oliveira, Maria Conceição de, 1996, Os curadores kaingang e a recriação de suas práticas: estudo de caso na aldeia Chapecó (oeste S. C.), tesis de maestría en antropología social, Universidade Federal de Santa Catarina, Florianópolis.

Oliveira, Geraldo Ferreira, Teresinha Regina Ribeiro de Oliveira, Fernanda França Rodrigues, Lincoln Ferreira Corrêa, Adauto Tsutomu Ikejiri y Luis Augusto Casulari, 2011, "Prevalência de diabetes melito e tolerância à glicose diminuída nos indígenas da Aldeia Jaguapiru, Brasil”, en Revista Panamericana de Salud Pública, vol. 29, núm. 5, pp. 315-321.

Oliveira, Philippe Hanna de Almeida, 2009, Comida forte e comida fraca: fabricação dos corpos e nutrição entre os kaingang da Terra Indigena Xapecó (Santa Catarina, Brasil), tesis de maestría en antropología social, Universidad Federal Santa Catarina, Florianópolis.

Portela, Sandra Carolina, 2010, Diabetes e hipertensão arterial entre indígenas kaingang de Terra Indígena Xapecó (SC): práticas de autoatenção em um contexto de intermedicalidade, tesis de maestría en antropología social, Universidad Federal Santa Catarina, Florianópolis.

Rocha, Ana Karina Silva da, Angelo José Gonçalves Bós, Edison Huttner y Denise Cantarelli Machado, 2011, "Prevalência da síndrome metabólica em indígenas com mais de 40 anos no Rio Grande do Sul, Brasil”, en Revista Panamericana de Salud Pública, vol. 29, núm. 1, pp. 41-45.

Salvo, Vera Lúcia Morais Antonio de, Douglas Rodrigues, Roberto G. Baruzzi, Heloisa Pagliaro y Suely Gimeno, 2009, "Perfil metabólico e antropométrico dos Suyá. Parque Indígena do Xingu, Brasil central”, en Revista Brasileira de Epidemiologia, vol. 12, núm. 3, pp. 458-468.

Schweikardt, J. y Luiza Garnelo, 2003, "Controle da hipertensão no Programa Médico de Família de Manaus e seus impasses qualitativos”, en Ciência \& Saúde Coletiva, vol. 8, supl. 1 y 2, pp. 91-99.

Souza, Maximiliano Loiola Ponte de (org.), 2013, Processos de alcoolização indígena no Brasil: perspectivas plurais, Fundação Oswaldo Cruz, Río de Janeiro.

Souza, Maximiliano Loiola Ponte de y Luiza Garnelo, 2006, "Desconstruindo o alcoolismo: notas a partir da construção do objeto de pesquisa no contexto indígena", en Reista Latinoamericana Psicopatologia Fundamental, vol. 9, núm. 2, pp. 279-292.

__ , 2007, "Quando, como e o que se bebe: o processo de alcoolização entre populações indígenas do alto Rio Negro, Brasil”, en Cadernos de Saúde Pública, vol. 23, núm. 7, pp. 1640-1648.

Veiga, Juracilda, 2000, Cosmologia e práticas rituais kaingang, tesis de doctorado en antropología social, Unversidade Estadual de Campinas, Campinas.

\section{Entrevistas}

Ana, Terra Indígena Xapecó, 20 de octubre de 2009.

Luciana, Terra Indígena Xapecó, 19 de septiembre y 20 de octubre de 2009.

Marino, Terra Indígena Xapecó, 30 de noviembre de 2009

Pedro, Terra Indígena Xapecó, 20 de octubre de 2009.

Rafael, Terra Indígena Xapecó, 30 de noviembre de 2009. 